\title{
Human Papilloma Virus Vaccine in Indian Settings: Need of the Hour
}

\author{
Akanksha Rathi ${ }^{1^{*}}$, Suneela Garg ${ }^{2}$ and GS Meena ${ }^{3}$ \\ ${ }^{1}$ Department of Community Medicine, Maulana Azad Medical College, Maulana Azad Medical College, Bahadurshah Zafar Marg, New Delhi, India \\ ${ }^{2}$ Director Professor, Department of Community Medicine, Maulana Azad Medical College, Bahadurshah Zafar Marg, New Delhi, India \\ ${ }^{3}$ Director Professor, Department of Community Medicine, Maulana Azad Medical College, Bahadurshah Zafar Marg, New Delhi, India
}

*Corresponding author: Akanksha Rathi, Department of Community Medicine, Maulana Azad Medical College, Maulana Azad Medical College, Bahadurshah Zafar Marg, New Delhi, 110002, India, Tel: +919911214187; E-mail:akanksharathi.dr@gmail.com

Received date: November 08, 2016; Accepted date: November 25, 2016; Published date: November 30, 2016

Copyright: (c) 2015 Rathi A, et al. This is an open-access article distributed under the terms of the Creative Commons Attribution License, which permits unrestricted use, distribution, and reproduction in any medium, provided the original author and source are credited.

\begin{abstract}
Cervical cancer is a major public health challenge accounting for a fifth of the global incidence and mortality. India has a population of 432.20 million women aged 15 years and above who are at risk of developing cervical cancer. The factors leading to high prevalence of cervical cancer in India are many. The high mortality due to the disease is mainly due to lack of awareness and absence of organized screening programs. India needs to take a leaf out of the book of 58 countries that have included Human Papilloma Virus (HPV) vaccine in their national immunization schedule so that the prevalence of this cancer can be brought down. Simultaneously, a need of organized screening programs throughout the country cannot be ignored.
\end{abstract}

Keywords: Human papilloma virus; Cervical cancer

\section{Cervical Cancer- A Public Health Menace}

Preventive health has been long neglected in our country and people still believe in rectifying what's broken rather than protecting what can be saved. Cervical cancer is one of the easily preventable cancer owing to long lag period, availability of screening method for its early detection and highly efficacious treatment [1]. The causal role of human papillomavirus infections in cervical cancer has been documented beyond reasonable doubt and the association is present in virtually all cervical cancer cases worldwide [2]. The commentary tries to throw light on the importance of the ignored but much needed HPV vaccine.

Cervical cancer is the fifth most common cancer of the world and third most common cancer in the women worldwide [3]. Cervical cancer is the 2nd leading cause of cancer deaths in women cancer deaths in women aged 15 to 44 years in India [4].

It is a major public health challenge accounting for a fifth of the global incidence and mortality. Mortality due to cervical cancer is higher in Indian woman than women of any other country due to late diagnosis as is evident from the fact that $85 \%$ of the cases present in advanced and late stages [4].

India has a population of 432.20 million women aged 15 years and above who are at risk of developing cervical cancer [5]. Current estimates indicate that every year 122, 844 women are diagnosed with cervical cancer and 67,477 die from the disease [6]. The morbidity burden of cervical cancer is also humongous. At a rate of 113 age adjusted Disability Adjusted Life year (DALY) per 100,000 populations, India accounts for $26.5 \%$ of global cervical cancer DALYs. Also, cervical cancer accounts for $11.6 \%$ of total cancer DALYs in India [7]. The financial burden that this disease poses over Indian economy is more than any other chronic disease with the exception of only cardiovascular disease. Due to high number of cervical cancer cases in the population, it has highest total cost of secondary care (100,000 INR per 100,000 populations) as compared to all other cancers [7].

The association of cervical cancer and Human Papilloma Virus (HPV) is well studied and documented [2,8-10]. The most common HPV types contributing to cervical cancer are HPV 16 \& 18. In India, these two types are associated with more than $70 \%$ of cervical cancer cases [11]. It is seen that $70 \%$ of sexually active individuals will attain one of the HPV genotypes at some point in their lives. Women in early adulthood, namely those under 25 years of age, have the highest rate of infection [12].

\section{Reasons for High Prevalence of HPV Infection in India}

HPV infection is the most common sexually transmitted infection worldwide. Persistent HPV infection is the necessary cause for development of cervical cancer and other exogenous or endogenous factors in conjunction with HPV infection influence the risk for cervical cancer. It is seen that among HPV positive women, high parity, long-term oral contraceptive use, smoking, and co-infection with other sexually transmitted agents are the most consistently identified environmental co-factors likely to influence the risk of progression from cervical HPV infection to High grade squamous intraepithelial lesion (HSIL) and Invasive Cervical Cancer [13].

The factors leading to high prevalence of cervical cancer in India are many. For e.g., genital hygiene standards are quite low. Of the 355 million menstruating women in India, only $12 \%$ use sanitary napkins. Women get married at an early age (average age 20 years according to National Family Health Survey-3), usually have no control over their fertility, consummate early and bear many children. Chlamydia infection is prevalent in India as the most important risk factor for acquiring this infection is consummating at young age. In a study done in New Delhi in 2013 it was seen that Chlamydia trachomatis was detected in $23.0 \%$ of patients attending gynecology OPD and $19.9 \%$ of patients attending STD clinic [14]. 


\section{Reasons for High Cervical Cancer Mortality in India}

Two major reasons for high mortality are no organized programs for cervical cancer screening, early detection and treatment of CIN and low awareness about cervical cancer.

The problem is not just the prevalence but also the high mortality due to this disease. Every 7 minutes, an Indian dies of cervical cancer. (WHO 2010) Survival rate is poor and less than 50\% women diagnosed with cervical cancer are able to survive for more than 5 years. The reason for such a high mortality due to cervical cancer is late diagnosis. Despite of the considerable burden, there are only few organized cervical cancer screening programs in the country. The majority of women are diagnosed only after they become symptomatic or at advanced stages of disease, with poor prognosis [3]. Screening of asymptomatic women is practically absent, even among otherwise well-organized health care programs of the industrial and military sectors [15]. It is estimated that less than 1.5 million smears are opportunistically taken annually. In recent years, HPV DNA testing is increasingly used in the private sector, though it is likely that less than 50,000 HPV tests are carried out annually [15].

Women have very low awareness about this disease and its prevention. Nearly half of Indian women (45\%) revealed that they worried more about getting obese than developing cervical cancer (24\%) [16]. A study on women from the metropolitan area of Chennai revealed that majority of women (69.6\%) were not aware of cervical cancer and very few (16.4\%) were aware of screening [17].

Both incidence and mortality rates increase when the human development is low and gender inequality levels are high [18]. Unfortunately, India fulfills both these conditions.

\section{HPV Vaccine and its Efficacy and Safety}

The need of vaccination arises as natural HPV infection induces a very weak immune response and may not lead to protection from reinfection [19-21]. On the other hand, the vaccine produces robust immune response. Two different vaccines that have been developed to prevent infection from HPV 16 and 18 and one of these offers added protection against HPV 6 and 11 (which cause genital warts) [12]. The quadrivalent and bivalent vaccines have been licensed for use in several countries [22]. Both vaccines need to be administered with 2 doses at 0 and 6 months prior to 15th birthday and 3 doses after $15^{\text {th }}$ birthday. They are being given to females aged 9-26 years, and are most effective if given before the female's first sexual encounter [7]. Health care providers worldwide have established unexposed young females as the primary target group for HPV vaccination [12]. The initiation of sexual activity has been found to be on average between the ages of 15-19 among women around the world [12]. In a survey among college students in Delhi, the age at sexual debut is earlier than the legal age at marriage, which is 18 year [19]. Similar results have been reported from the National Family Health Survey also. In order to ensure that recipients receive maximum protection, the target population should be young adolescents (9-13 years of age). Also, at younger age the recipients mount a better immune response $[19,23]$.

In randomized double blind studies, the bivalent vaccine was found to have $87.5 \%$ efficacy in preventing persistent infection due to HPV $16 / 18$ and $92 \%$ [9] efficacy in preventing HPV 16/18 associated cytological abnormalities on intention-to-treat analysis [24]. Intentionto-treat analysis of the efficacy of the quadrivalent vaccine observed that the vaccine was $88 \%$ effective at preventing persistent infection and $100 \%$ effective in preventing histologically confirmed cervical disease associated with HPV 6/11/16/18 [24].

Since first being licensed at the beginning of 2006, more than 200 million doses of HPV vaccines have been distributed globally [25]. WHO recommends introduction of HPV vaccine in national immunization programs provided that the introduction is feasible, cost-effective, sustainable and cervical cancer is a public health priority; and to date, it has not found any safety issue that would alter its recommendations for the use of the vaccine [25].

\section{Effectiveness of HPV Vaccine School Campaigns}

The vaccines have been introduced in school immunization programs by many developed and developing countries. Countries like UK, Ireland, Scotland, Malaysia and Canada claim more than $80 \%$ HPV Vaccine coverage amongst adolescent schoolgirls. African countries like Zambia, Rwanda, Kenya, Laos, Uganda \& Ghana have also introduced HPV vaccine in school immunization programs with the help of Global Alliance for Vaccination and Immunization (GAVI).

Australia's HPV vaccination program is one of the most successful in the world, started in 2007, offers free HPV vaccination to girls who are 12 and 13 years old and catch-up programs for girls and women under 26. A study by Donovan et al found that diagnoses of genital warts among young women ages 12 to 26 plummeted 59\%, genital warts in males in the same age group dropped $39 \%$ and there was also a striking decline in the rate of high-grade cervical abnormalities in teenage girls, a sign that a decline in cervical cancer cases may be on the horizon, in the two years after the program began [6]. Australia now has the lowest cervical cancer prevalence in the world.

\section{Rationale of Introducing HPV Vaccine in a Country like India}

Goldie et al estimated health and economic estimation of HPV 16 and 18 genotype vaccination in 72 GAVI-eligible countries (India being one of them) [26]. Using the present data from these countries, they estimated cervical cancer cases, deaths and DALYs averted and costs saved. In countries like India, Uganda and Kenya, if more than $70 \%$ of the target populations (adolescent girls) are vaccinated then mean reduction in the lifetime risk of cancer will be more than $50 \%$. Also, the vaccination was found to be highly cost-effective when DALYs averted were calculated. Additionally, a 10-year modeled scenario showed that the future deaths of approximately 2 million women vaccinated as adolescents would be prevented. A qualitative systematic review by Marra et al. [27], did effectiveness or cost effectiveness analyses of an HPV vaccine compared with the current cytology-based Pap smear screening program. Despite of their heterogeneity, all studies showed that vaccination would decrease rates of HPV infection, precancerous lesions and cervical cancer and a female-only vaccination program is cost effective as compared with the current cytology-based Pap smear screening program. So, in India where the screening infrastructure is nascent or infantile, a vaccination program offers a cost-effective choice.

\section{India Lags Behind}

The quadrivalent vaccine was the first to be licensed in 2006 and is available in the US, Europe, Australia and Asia, in over 100 countries. The bivalent vaccine was introduced in 2007 and is available in nearly 80 countries. In India, both vaccines are licensed to be given in females 
(in some countries they are licensed to be given in both males and females) [23]. By August 2014, 58 countries (30\%) had introduced HPV vaccine in their national immunization program for girls. Even other Southeast Asian countries like Malaysia, Hong Kong \& Singapore have an effective cervical cancer-screening program and HPV immunization in place. In a welcome move, the government of Delhi recently announced introduction of HPV vaccine for grade 5 girls studying in government \& government-aided schools. However, other states are still to introduce the same. Indian government is still struggling to improve the immunization status of under-5 children and vaccination of adolescent girls is nowhere on its priority list.

The advisory bodies in India like Indian academy of pediatrics (IAP) and federation of obstetric and gynecological societies of India (FOGSI) have recommended HPV vaccination. However, only few young girls have access to the vaccine since the vaccine is costly and is primarily available with the private sector (Gardasil-3000 INR per shot \& Cervarix-2000 INR per shot).

The awareness about the vaccine even among high socio-economic groups is quite low. A survey in Eastern India among educated urban men and women, with at least one girl child and belonging to middle or high socio-economic group, revealed that $72 \%$ had never heard of HPV. Only $46 \%$ of parents were in favor of vaccinating their daughters against an STI; however, after going through a brief information sheet about the HPV vaccine, $80 \%$ agreed to vaccination. The most common reason for not accepting the vaccine was uncertainty about the safety of a new vaccine. The physician's recommendation was found to be the most important factor influencing their decision [15]. Major determinants of social acceptance of vaccine in India are parental awareness and attitude [19]. A study done in college girls in Kolkata revealed that though the knowledge of girls about screening methods was low but a majority desired to have protective vaccination against cervical cancer [28].

A research paper by Tsui et al. has listed a few reasons for the delays in new vaccine adoption in a resource-limited country. The reasons are as follows [29].

- Financial constraints \& political obstacles interfering with market forces

- Competing health priorities

- Absence of national disease burden data illustrating magnitude of problem

- Lack of country specific vaccine efficacy data

- Concern about sustainable supply for new vaccine

In Indian context, financial constraints are there and so are competing health priorities like immunization of under-5 children. However, there is abundant data illustrating the magnitude of cervical cancer problem so much so that India shares one-fifth burden of cervical cancer mortality of the world. Since the vaccine has not been given in large cohorts and the private sector has given this vaccine discreetly, there is a definite lack of specific vaccine efficacy data. The concern about sustainable supply of the vaccine is also real. The other issues could be the international licensing and technology transfer as well as cGMP manufactures capacity. Thus all these factors are responsible for the doubts that Government of India has about introducing this vaccine.

\section{Recommendations-Need of the Hour}

India with its highest share of global burden of cervical cancer has to implement a population based cervical cancer control program to reduce the number of deaths. A recent report by WHO states that lowand middle-income countries, where more than $85 \%$ of cervical cancer deaths occur, can particularly benefit from HPV vaccine [30]. Human papillomavirus (HPV) vaccine has one of the highest per-person impacts on mortality of all vaccines. In the longer-term, high HPV vaccine coverage will reduce the economic and human costs of cervical cancer. Moreover, in Indian settings where women have less access to cervical cancer screening the vaccine is particularly beneficial [30].

Regular cervical cytology examination (Pap smear) by all women who have initiated sexual activity can prevent the occurrence of cervical cancer. The primary target should be to offer once a lifetime screening for all women at the age of 40 years. Government and private health care providers can join in this effort and offer these services [31]. Though cytological examination has been the mainstay for early detection of cervical cancer, its widespread use is not possible in our country due to paucity of resources, manpower and other facilities [32]. The patient has to be followed up for intervention and screening might still be easier than follow-up.

Alternative strategies such as naked eye visual inspection of cervix (down staging), visual inspection with acetic acid (VIA), magnified VIA (VIAM), visual inspection with lugol's iodine (VIAL), cervicography and HPV DNA testing in detecting cervical cancer and its precursors have to be adopted [33]. Regular screening through VIA has along with further referral for treatment has been suggested in a pooled analysis [34]. As the age affected by cervical cancer is decreasing thus a study has suggested that screening should be initiated at 25 years of age [19]. Screening approaches in India and other developing countries can reduce the lifetime risk of cancer by approximately $25-36 \%$ [15].

A national HPV vaccination program appears to be practically possible as compared to screening program in India. The infrastructure and trained personnel for vaccination are already in place at all levels of health service delivery [32]. Policy makers should realize the importance of this vaccine and should seriously consider including HPV vaccine in National Immunization Schedule.

The vaccine is creating a buzz in the private sector. Efforts should be made to increase the awareness about this disease so that the unfelt need of the society can be converted into felt need. A vaccine program cannot be successful without the support and approval of the general public so media should be very responsible. Role of press should be supportive \& adverse effects following immunization (AEFI) should not be misreported \& blown out of proportion.

\section{References}

1. Cervical cancer is preventable (2014) Center for Disease Control and Prevention.

2. Bosch FX, Lorincz A, Muñoz N, Meijer CJ, Shah KV (2002) The causal relation between human papillomavirus and cervical cancer. J Clin Pathol 55: 244-265.

3. Krishnan S, Madson E, Porterfield D, Varghese B (2013) Advancing cervical cancer prevention in India : insights from research and programs. Health, Nutrition and Population (HNP) discussion paper. Washington, DC; World Bank Group.

4. Sreedevi A, Javed R, Dinesh A (2015) Epidemiology of cervical cancer with special focus on India. Int J Womens Health 7: 405-414. 
5. Trivedi V, Kumar R, Rani R, Kumari A, Chauhan R (2015) Cervical cancer awareness in Bihar, India: Lots of light but no illumination. IOSR Journal of Dental and Medical Sciences 14: 88-92.

6. Nath A, Kumar R, Trivedi V, Murti K, Kumar S, et al. (2015) MDA Elevation and Hematological Derangements at First Clinical Presentation in Cervical Cancer Patients. Int J Pharm Sci Rev Res 30: 175-179.

7. Satija A (2009b) Cervical cancer in India, South Asia center for chronic disease WHO. GLOBOCON 2002 (IARC), pp: 6-26.

8. Kjaer SK, van den Brule AJC, Paull G, Svare EI, Sherman ME, et al. (2002) Type specific persistence of high risk human papillomavirus (HPV) as indicator of high grade cervical squamous intraepithelial lesions in young women: population based prospective follow up study. Br Med J 325: 572-575.

9. Schlecht NF, Kulaga S, Robitaille J, Ferreira S, Santos M, et al. (2001) Persistent human papillomavirus infection as a predictor of cervical intraepithelial neoplasia. JAMA 286: 3106-3114.

10. Walboomers JMM, Jacobs MV, Manos MM, Bosch FX, Kummer JA, et al. (1999) Human papillomavirus is a necessary cause of invasive cervical cancer worldwide. J Pathol 189: 12-19.

11. HPV Centre (2014) Human Papilloma virus and related disease report, India. ICO, Barcelona, Spain.

12. Munoz K, Shroff N, Brown B (2012) Reducing HPV Transmission in Developing Countries: The Importance of Education and the Necessary Vaccinations.

13. Castellsagué X, Bosch FX, Muñoz N (2002) Environmental co-factors in HPV carcinogenesis. Virus Res 89: 191-199.

14. Malhotra M, Sood S, Mukherjee A, Muralidhar S, Bala M (2013) Genital Chlamydia trachomatis: An update. Indian J Med Res 138: 303-316.

15. Senapathy JG, Umadevi P, Kannika PS (2011) The Present Scenario of Cervical Cancer Control and HPV Epidemiology in India: an Outline. Asian Pacific J Cancer Prev 12: 1107-1115.

16. Express News Service (2012) Cervical Cancer: Aware but busy. The New Indian Express.

17. Beining RM (2012) Screening for cervical cancer: an exploratory study of urban women in Tamil Nadu, India.

18. Singh GK, Azuine RE, Siahpush M (2012) Global Inequalities in Cervical Cancer Incidence and Mortality are Linked to Deprivation, Low Socioeconomic Status, and Human Development. Int J MCH AIDS.1: 17-30.

19. Nigam A, Saxena P, Acharya AS, Mishra A, Batra S (2014) HPV Vaccination in India: Critical Appraisal. ISRN Obstetrics and Gynecology.
20. Carter JJ, Koutsky LA, Hughes JP, Lee SK, Kuypers J, et al. (2000) Comparison of human papillomavirus types 16, 18, and 6 capsid antibody responses following incident infection. J Infect Dis 181: 1911-1919.

21. Schwarz TF, Leo O (2008) Immune response to human papillomavirus after prophylactic vaccination with AS04-adjuvanted HPV-16/18 vaccine: improving upon nature. Gynecol Oncol. 110: S1-10.

22. Human papillomavirus vaccines (2014) WHO position paper. Geneva: World Health Organization. Report no. 43.

23. Bhatla N, Joseph E (2009) Cervical cancer prevention \& the role of human papillomavirus vaccines in India. Indian J Med Res 130: 334-340.

24. Villa LL (2006) Prophylactic HPV vaccines: reducing the burden of HPVrelated diseases. Vaccine 24 Suppl 1: S23-28.

25. Global Advisory Committee on Vaccine safety Statement on Safety of HPV vaccines (2015). Geneva: World Health Organization.

26. Goldie SJ, O'Shea M, Campos NG, Diaz M, Sweet S, et al. (2008) Health and economic outcomes of HPV 16,18 vaccination in 72 GAVI-eligible countries. Vaccine 26: 4080-4093.

27. Marra F, Cloutier K, Oteng B, Marra C, Ogilvie G (2009) Effectiveness and cost effectiveness of human papillomavirus vaccine: a systematic review. Pharmacoeconomics 27:127-47.

28. Saha A, Chaudhury AN, Bhowmik P, Chatterjee R (2010) Awareness of Cervical Cancer Among Female Students of Premier Colleges in Kolkata, India. Asian Pacific J Cancer Prev 11: 1085-1090.

29. Tsui J, LaMontagne DS, Levin C, Bingham A, Menezes L (2009) Policy Development for Human Papillomavirus Vaccine Introduction in LowResource Settings. The Open Vaccine Journal 2:113-122.

30. HPV vaccine communication (2013) Special considerations for a unique vaccine. Geneva: WHO.

31. Varghese C (2015) Cancer Prevention and Control in India.

32. Basu P, Chowdhury D (2009) Cervical cancer screening \& HPV vaccination: a comprehensive approach to cervical cancer control. Indian J Med Res 130: 241-246.

33. Murthy NS, Mathew A (2004) Cancer epidemiology, prevention and control. Curr Sci 86: 518-527.

34. Patra J, Dikshit R, Bhatia M, Ramasundarahettige CC, Jha P (2015) HPVavertable cancer risks in India: A pooled analysis of 9 observational studies. Int J Cancer 136: 491-492. 